\title{
FACTORES HUMANOS EN AVIACIÓN: CRM (CREW RESOURCE MANAGEMENT - GESTIÓN DE RECURSOS DE LA TRIPULACIÓN) HUMAN FACTORS IN AVIATION: CRM (CREW RESOURCE MANAGEMENT)
}

\author{
Daniel Muñoz-Marrón \\ Piloto del Ejército del Aire (Ministerio de Defensa). Psicólogo Especialista en Psicología Clínica
}

Uno de los campos aplicados a los que más ha contribuido la ciencia psicológica es, sin lugar a dudas, el de la aviación. El análisis y estudio de los factores humanos constituye actualmente uno de los puntos fuertes en el sector aeronáutico de cara a la reducción de los accidentes aéreos. Desde su aparición en 1979, los programas de Gestión de Recursos de la Tripulación (CRM) han sido una de las herramientas que con mayor éxito han gestionado el denominado "error humano". El presente artículo realiza un breve recorrido por la historia de estos programas globales de entrenamiento que suponen uno de los grandes logros de la Psicología Aplicada.

Palabras clave: Gestión de Recursos de la Tripulación (CRM), Entrenamiento de vuelo, Factores humanos, Psicología de la Aviación, Seguridad Aérea.

One of the applied fields to which psychological science has most contributed is, without doubt, aviation. The analysis and study of human factors is currently one of the strong points in the aeronautical sector in order to reduce accidents in aviation. Since its appearance in 1979, the Crew Resource Management (CRM) programs have been one of the most successful tools that have managed the so-called "human error". This paper makes a brief tour through the history of these global training programs that represent one of the great achievements of Applied Psychology.

Key words: Crew Resource Management (CRM), Flight training, Human factors, Aviation Psychology, Aviation Safety.

L a aviación se ha convertido en uno de los pilares básicos sobre los que se asienta la vida social y la actividad económica, y la Psicología ha contribuido de forma notable a este desarrollo. La psicología aplicada apareció como respuesta a las demandas del gran cambio social y tecnológico que se produjo en las últimas décadas del siglo XIX y comienzos del siglo XX (Sáiz y Sáiz, 2012), y la aviación se convirtió en uno de los campos más interesantes de aplicación de la ciencia psicológica a cuestiones de carácter práctico (Dockeray e lsaacs, 1921).

La I Guerra Mundial fue el detonante que desencadenó la introducción de la Psicología en el mundo de la aviación, centrándose inicialmente en la búsqueda de las características que debía poseer el aviador "ideal" (Muñoz-Marrón, en realización). Esta etapa inicial se caracterizó por la realización de pruebas y exámenes psicológicos de aptitud (Baumgarten, 1957) y personalidad, cuyo objetivo era conocer qué cualidades específicas debían poseer los pilotos (Sáiz y Sáiz, 2012). Estos primeros estudios y trabajos mezclaban aspectos más cercanos a la fisiología con aquellos de ámbito puramente psicológico.

Recibido: 12 enero 2018 - Aceptado: 8 junio 2018

Correspondencia: Daniel Muñoz-Marrón. Piloto del Ejército del Aire (Ministerio de Defensa).Psicólogo Especialista en Psicología Clínica. 43 Grupo de Fuerzas Aéreas. Base Aérea de Torrejón. Autovía A2 s/n. 28850 Torrejón de Ardoz. Madrid. España. E-mail:dmunmal@ea.mde.es
El periodo de entre guerras se caracterizó por un descenso en el interés de los investigadores por la aplicación de la ciencia psicológica a las fuerzas armadas, y en concreto al arma aérea, al desaparecer la urgencia en la necesidad de la selección de pilotos.

En el campo psicológico, la II Guerra Mundial supuso un avance en el estudio de la selección y el entrenamiento de los aviadores, provocado por la cada vez más difícil adaptación de éstos a máquinas más complejas y veloces (Alonso, 1997). Se introducen evaluaciones de los entrenamientos y se evoluciona desde el abordaje de aspectos más cognitivos y motrices hacia otros de carácter motivacional. Terminada la contienda, el objetivo de la investigación cambia radicalmente, pasando de un ámbito específicamente bélico hacia otro en el que prima la investigación civil. Esto provoca que en 1949 las compañías aéreas más punteras comiencen a contratar a los primeros psicólogos (Alonso, 1997).

A finales de los años setenta se produce un hecho que cambiará de un modo radical la relación entre la ciencia psicológica y la aviación (Muñoz-Marrón, en realización). Tal y como se describirá más adelante, en un encuentro auspiciado por la Administración Nacional de la Aeronáutica y del Espacio de los Estados Unidos (National Aeronautics and Space Administration [N.A.S.A.]) aparece el concepto de Gestión de Recursos de Cabina (Cockpit Resource Management [CRM]) como programa de entrenamiento de las tripulaciones aéreas, que tras varias etapas de desarrollo llega a nuestros días como Gestión 
de Recursos de la Tripulación (Crew Resource Management) (Helmreich, Merritt y Wilhelm, 1999). El CRM supone una nueva forma de trabajo en equipo para las tripulaciones aéreas y se refiere básicamente al manejo óptimo de los recursos disponibles en términos de tecnología de la aeronave, tripulación de vuelo, cabina de pasajeros, control de tráfico aéreo, ayudas a la navegación, etcétera (Turner, 1995).

\section{¿QUÉ ES EL CRM?}

Diseñado para reducir el error e incrementar la efectividad de las tripulaciones aéreas (Wiener, Kanki y Helmreich, 1993), el CRM puede definirse como la óptima utilización, por parte de una tripulación, de todos los recursos disponibles (información, equipos materiales y recursos humanos) para la consecución de operaciones de vuelo seguras y eficientes (Lauber, 1984). Engloba un conjunto de comportamientos y estrategias que posee y ha de seguir, en aras de la seguridad, el equipo que conforma una tripulación de vuelo (Helmreich y Foushee, 1993), y constituye el reconocimiento formal por parte del sector aeronáutico de la importancia que tiene el estudio de los factores humanos en la optimización de las operaciones aéreas y la seguridad de vuelo

Utilizado tanto en aviación comercial como militar, el CRM es en realidad un programa global de formación y entrenamiento en habilidades y capacidades de gestión, basado en evidencias, y diseñado para mejorar la comunicación, la toma de decisiones y la adaptación entre miembros de un equipo en situaciones críticas (Salas, Burke, Bowers y Wilson, 2001; Shuffler, DiazGranados y Salas, 2011); una herramienta para combatir aquellos accidentes en los cuales un deficitario trabajo en equipo en la cabina de vuelo podría suponer un factor precipitante de los mismos (Salas, Wilson y Edens, 2009). El objetivo del programa es el de combinar habilidades técnicas y humanas para lograr operaciones aéreas más seguras y eficaces (Federal Aviation Administration [F.A.A.], 2012).

Resulta ser un método creado para optimizar el rendimiento reduciendo el efecto de los errores humanos a través del uso de todos los recursos para resolver los problemas, incluyendo personas, tecnología y procesos (Marshall, 2010). De forma más detallada, el propio Marshall (2010) basa su definición del programa en tres pilares fundamentales que sintetizamos a continuación:

$\checkmark$ Un enfoque de sistemas para la seguridad, que hace hincapié en la naturaleza inherente del error, promoviendo una cultura no punitiva y centrándose en procedimientos concretos y normalizados de trabajo.

v Un sistema integral basado en la práctica y operacionalmente dirigido a la aplicación proactiva de los factores humanos para mejorar el rendimiento del equipo.

$\checkmark$ Un sistema caracterizado por: (1) Definir a la tripulación en su conjunto, en lugar de al individuo, como unidad de formación estándar; (2) centrarse en cómo las actitudes de los miembros de la tripulación y sus comportamientos afectan a la seguridad; (3) emplear una metodología de formación activa y práctica, basada en la participación y el aprendizaje mutuo; (4) incluir técnicas de liderazgo, habilidades de trabajo y manejo de equipos; (5) promover la creación de equipos de trabajo participativos, preservando la autoridad y la cadena de mando; $y$, (6) proporcionar a individuos y equipos la oportunidad de revisar y analizar su propio desempeño e introducir las mejoras apropiadas.

Los programas de CRM hacen hincapié en la naturaleza del error en la medida en que reconocen que el error es inherente a la conducta humana. Para reducir al máximo su aparición, se crean procedimientos de trabajo muy definidos, al tiempo que se dota a las tripulaciones de métodos para reportar libremente los errores cometidos, sin que estos lleven aparejada una sanción. Comunicaciones que contribuyen a crear un sistema proactivo de mejora del rendimiento, en la medida en que intenta crear "barreras frente al error" antes de que éste ocurra. Tomando al equipo como unidad de actuación, y mediante la utilización de una metodología práctica, basada en la participación activa y el aprendizaje mutuo, el programa busca modificar actitudes y crear verdaderos equipos de trabajo en los que, sin dañar la cadena de mando, los participantes puedan analizar su propio desempeño e introducir las mejoras pertinentes con el objetivo de optimizar el rendimiento y lograr operaciones de vuelo más seguras.

Los programas de entrenamiento en CRM son, en definitiva, una herramienta fundamental creada para mejorar el rendimiento de un equipo con el objetivo de reducir el efecto del denominado "error humano" y optimizar la ejecución a través de la utilización de todos los recursos disponibles.

La aparición del CRM constituyó un hito clave en la relación Psicología-Aviación y provocó un cambio radical en el estudio de la seguridad aérea, gracias al cual la ciencia psicológica se convierte en pilar esencial en la búsqueda de la seguridad de vuelo y un aliado de la industria de la aviación en el desarrollo de los programas de capacitación dirigidos a reducir los errores humanos y aumentar la eficacia de las tripulaciones de vuelo.

Por esta razón, el CRM constituye una de las historias de éxito de la psicología moderna y la ingeniería cognitiva (Cooke y Durso, 2007) y una herramienta de prevención esencial en el actual mundo de la aviación (Maurino, 1999), creada desde el mundo de la Psicología. Las estrategias de CRM se utilizan en la actualidad con el objetivo de mejorar la seguridad, reduciendo el error humano y proporcionando a las tripulaciones el entrenamiento necesario que les capacite para hacer uso de todos los recursos a su alcance (Salas y Cannon-Bowers, 2001).

En la actualidad, los programas de CRM incluyen diferentes módulos que abordan conceptos clave a partir de un diseño que contempla tres grandes áreas de trabajo: Command (mando y dirección, procesos de comunicación y toma de decisiones), leadership (liderazgo, clima de trabajo y gestión del equipo) y resource management (gestión de recursos, carga de trabajo y análisis de la situación). 
Los cursos introductorios de CRM, que realizan las diferentes compañías aéreas y las unidades de la fuerza aérea de los ejércitos, tienen generalmente una duración de entre dos y cinco días, y están dirigidos por psicólogos y pilotos que trabajan conjuntamente en su desarrollo. Los métodos de enseñanza incluyen lecturas, ejercicios prácticos, role playing, estudio de casos y videos de recreación de accidentes $\left(\mathrm{O}^{\prime}\right.$ Connor y Flin, 2003), entre otras técnicas de trabajo. Aunque no existe una metodología estandarizada para su desarrollo (Salas, Fowlkes, Scout, Milanovich y Prince, 1999), los cursos habitualmente abordan temáticas tales como trabajo en equipo, liderazgo, conciencia situacional, toma de decisiones, comunicación y limitaciones personales (Flin y Martin, 2001), aunque la variabilidad al respecto es importante, debido fundamentalmente a que su diseño se adapta a las necesidades del operador aéreo que los lleva a cabo.

\section{ORIGEN DEL CRM}

Una serie de accidentes ocurridos en aviación en la década de 1970 (Helmreich et al., 1999), así como la alta incidencia de casos en que tales desastres fueran debidos al error humano, provocaron la implantación de los programas de entrenamiento en CRM. La N.A.S.A., ya pionera en el estudio de los factores humanos en la aeronáutica y astronáutica, inició su programa en factores humanos para la seguridad aérea en 1973 (Marshall, 2010). Desde su Ames Research Center (Moffett Field, CA) los investigadores, Charles K. Billings y John K. Lauber, y el piloto de pruebas, George E. Cooper, comenzaron a investigar preguntando directamente a los pilotos que se habían visto involucrados en algún accidente, intentando obtener así información detallada de su versión de los acontecimientos. El objetivo era elaborar un programa de investigación encaminado a analizar los errores humanos que podían estar detrás de los accidentes e identificar aquellos factores que estaban presentes en los denominados accidentes por "error del piloto" (Alkov, 1989).

Este programa de entrevistas condujo a la creación del Sistema de Reportes de Seguridad Aérea (Aviation Safety Reporting System [A.S.R.S.]) de la N.A.S.A. (Alkov, 1989; Amezcua Gonzalez, Lareo y Amezcua Pacheco, 2001). A partir de sus testimonios, los pilotos dejaron claro que los programas de entrenamiento no estaban a la altura de las necesidades que se presentaban durante el vuelo. Lo realmente significativo, por lo que a la Psicología se refiere, fue que su disconformidad no se refería a las técnicas de entrenamiento de vuelo, sino fundamentalmente a aspectos directamente relacionados con el comportamiento humano, haciendo especial hincapié en la toma de decisiones, el liderazgo y las comunicaciones interpersonales. Los investigadores comprobaron que para ser un buen piloto no bastaba con poseer una buena destreza a los mandos de una aeronave. Ruffel-Smith analizó en un simulador de vuelo el comportamiento de diferentes tripulaciones tanto en situaciones rutinarias como en situaciones de emergencia, y demostró que cuanto mejor era la utilización de los recursos por parte de la tripulación y más eficaz la comunicación entre sus miembros, mejor era la ejecución (Ruffel-Smith, 1979). Las conclusiones de diversas investigaciones permitieron identificar algunos indicios para elaborar posteriormente lo que hoy conocemos como entrenamiento en CRM.

\section{IMPORTANCIA HISTÓRICA Y DESARROLLO DEL CRM}

El entrenamiento en CRM, con las variantes que ha ido presentando a lo largo del tiempo, tiene su origen en junio de 1979 (F.A.A., 2012; Helmreich et al., 1999; Marshall, 2010; McKeel, 2012), concretamente en un encuentro realizado bajo el patrocinio de la N.A.S.A. (Alkov, 1989) y titulado Resource Management on the Flightdeck (Cooper, White y Lauber, 1980; Helmreich, 2006). Este evento se organizó, en parte, provocado por el accidente sufrido por el vuelo 173 de United Airlines, en diciembre de 1978, que la Junta Nacional de Seguridad en el Transporte (National Transportation Safety Board [N.T.S.B.]) de los Estados Unidos (EE.UU.) atribuyó fundamentalmente a un error del comandante de la aeronave al no atender las indicaciones de su tripulación, así como a la falta de asertividad por parte de ésta (N.T.S.B., 1978). El accidente constituye un claro ejemplo de gestión deficiente de los recursos humanos en cabina y de pésimo trabajo en equipo ante una situación de fallo en los sistemas de la aeronave, provocado fundamentalmente por la utilización de un estilo de liderazgo autoritario por parte del comandante de la aeronave.

Durante este encuentro, la N.A.S.A. presentó los resultados de la investigación que había realizado sobre las causas que subyacían a los accidentes en el transporte aéreo. Dichos resultados incluían la identificación de aquellos aspectos del error humano presentes en la mayoría de los accidentes aéreos, tales como fallos en la comunicación interpersonal o errores en la toma de decisiones y la gestión del liderazgo. Fue en ese momento cuando se acuñó el término de CRM para definir el programa de entrenamiento encaminado a reducir el error del piloto a partir de un mejor uso de los recursos humanos en la cabina de vuelo.

En este primer momento, las siglas CRM hacían referencia a Cockpit Resource Management, que poco después (finales de los años ochenta) pasarían a definir Crew Resource Management, sustituyendo el término Cockpit (cabina) por el de Crew (tripulación) y trasladando el foco de atención desde el piloto como elemento individual al de la tripulación como elemento grupal o de equipo de trabajo.

A comienzos de los años ochenta, la investigación sobre las causas de los accidentes de aviación permite la introducción de programas estructurados de entrenamiento en CRM en la aviación civil (Helmreich, 2006). En enero de 1981, la compañía United Airlines crea el primer entrenamiento específico en CRM (Helmreich et al., 1999). Después serían KLM en Europa y Ansett en Oceanía (Helmreich, 2006). Desde entonces, las aerolíneas más importantes, la Fuerza Aérea, el Ejército, la Ar- 
mada y la Guardia Costera de los EE.UU. han desarrollado y utilizado programas de CRM, si bien en algunos casos con otra nomenclatura (Alkov, 1989). Estos programas de entrenamiento no han dejado de extenderse, primero en los EE.UU. y posteriormente por todo el mundo, al tiempo que su utilización se ha generalizado a otros ámbitos laborales, tanto propios de la aviación, tales como el mantenimiento (Taylor y Thomas, 2003) o el control del tráfico aéreo, como ajenos a ella, como la medicina (Gordon, Mendenhall y O'Connor, 2013), la cirugía (Helmreich, 2006), las plataformas petrolíferas (O'Connor y Flin, 2003), el sector ferroviario (Sebastián, 2002, 2009), el de la fuerza submarina (Acuña, 2013) o el de los buceadores militares (O'Connor y Muller, 2006).

El proceso seguido por el CRM ha sufrido una evolución que le ha hecho pasar por diferentes etapas con características propias. Siguiendo a Helmreich (2006) y Marshall (2010), podemos distinguir seis grandes etapas en el desarrollo de los programas de formación en CRM, cada de ellas basada en los éxitos y lecciones aprendidas de la anterior. Resumimos a continuación los aspectos psicológicos más significativos que se contemplan en cada etapa, reflejando en cada caso las ideas de los autores citados.

\section{Primera generación: Gestión de Recursos de Cabina (Cockpit Resource Management)}

El programa iniciado por United Airlines en el año 1981 (Helmreich et al., 1999) fue denominado Gestión de Recursos de Mando y Liderazgo (Command-Leadership-Resource Management [C.L.R.]) (Helmreich, 2006). Consistía en una serie de seminarios durante los cuales los participantes analizaban sus propios estilos de gestión. Se utilizaron las aportaciones realizadas para la N.A.S.A. por Blake y Mouton, pioneros en consultoría de gestión, cuya obra adquirió relevancia en el campo de la Psicología del Trabajo y de las Organizaciones, hasta el punto de que su teoría, conocida como el managerial grid (Blake y Mouton, 1964, 1985) aparece en cualquier manual que se precie. El managerial grid, grid gerencial o rejilla de dirección consiste en una matriz numérica para identificar los diferentes estilos de liderazgo, en función de las dos dimensiones que se consideran fundamentales: el interés por las personas y el interés por la producción o los resultados. La teoría de estos autores considera que el estilo óptimo de dirección combina de manera armónica la preocupación por las personas y por los resultados, continuando con la ya entonces famosa Teoría Y, de McGregor (1960), obra clave de la Psicología Organizacional.

Pronto otras compañías aéreas realizaron programas similares centrados en modificar aquellos estilos diagnosticados como erróneos y corregir deficiencias asociadas a comportamientos individuales, como podía ser la muestra de autoridad excesiva por parte de los comandantes de aeronave sobre el resto de la tripulación, o la falta de asertividad que presentaban los pilotos con menor experiencia frente al comportamiento autoritario de sus comandantes.
Las reacciones a estos programas de primera generación fueron razonablemente positivas (Helmreich, 2006), aunque algunos pilotos ofrecieron ciertas reticencias a los mismos, acusándolos de intentar manipular sus personalidades (Helmreich et al., 1999) y mostrando fundamentalmente cierto miedo potencial a perder el control de las cabinas de vuelo por la intromisión de otros profesionales externos, fundamentalmente psicólogos, ajenos hasta entonces a la aviación.

\section{Segunda generación: Gestión de Recursos de la Tripulación (Crew Resource Management)}

En mayo de 1986, la N.A.S.A., que había estado implicada en el desarrollo del CRM desde sus inicios, organizó un encuentro para la industria aeronáutica (Helmreich, 2006; Orlady y Foushee, 1987) que se convirtió en el lugar ideal para la comunicación y posterior análisis de los resultados obtenidos por los operadores aéreos en sus primeras aproximaciones al CRM. Las conclusiones apuntaban a la modificación de los programas de CRM, de tal modo que éstos debían dejar de ser un elemento aislado en la formación de las tripulaciones para convertirse en componente esencial de un programa de entrenamiento más complejo que, realizado de forma periódica, combinara la formación teórica con sesiones prácticas en simulador de vuelo, denominadas L.O.F.T. (Line Oriented Flight Training) (Helmreich et al., 1999), en las que debían entrenarse las habilidades interpersonales abordadas en el aula.

Los programas de esta segunda generación incorporan la aplicación práctica de diferentes conceptos a la dinámica de trabajo que se produce entre los componentes de la tripulación, tales como dinámicas de grupo en cabina, orientadas fundamentalmente hacia el trabajo de la tripulación como equipo, $y$ al entrenamiento en estrategias de toma de decisiones o cómo romper la cadena del error. Los conceptos manejados hasta entonces se hacen más operativos, acercando de este modo los entrenamientos a las operaciones aéreas reales. Gracias, en parte, a esto último, la aceptación de estos programas por parte de las tripulaciones fue mayor que la que habían tenido los programas pertenecientes a la primera generación, eliminando gran parte de las resistencias que las tripulaciones manifestaban hacia la intervención de la Psicología en su formación.

Tercera generación: Los programas de entrenamiento en CRM extienden su alcance

Los programas de CRM adquieren tal relevancia que su utilización se extiende de un modo imparable. El CRM empieza a tener en cuenta los sistemas utilizados en aviación (Salas et al., 2001) y su diseño comienza a incorporar características propias del sector aéreo, con lo que se produce un gran avance en el entrenamiento específico de las tripulaciones de vuelo, al tiempo que se supera una de las críticas más significativas de los modelos de generaciones anteriores. Los esfuerzos se dirigen ahora hacia la integración del CRM con el entrenamiento 
técnico, poniendo especial atención en aquellas habilidades y comportamientos específicos que las tripulaciones podrían realizar durante el desarrollo de cualquier vuelo real para conseguir una ejecución más eficaz.

Durante estos años la industria aeronáutica evoluciona a pasos agigantados, mejorando de manera sustancial el diseño de las aeronaves, al tiempo que los aviones aumentan su fiabilidad (Alkov, 1989). Las cabinas de vuelo de los aviones sufren grandes modernizaciones con la inclusión de sistemas cada vez más seguros a la vez que complejos, con lo que la necesidad de lograr un correcto acoplamiento de las tripulaciones a las nuevas cabinas se vuelve fundamental. Por esta razón, muchas aerolíneas comienzan a incluir módulos de CRM diseñados de un modo específico para la correcta utilización de los diferentes elementos de las cabinas de vuelo de sus modernos aparatos. Los programas de entrenamiento también comienzan a adentrarse en otros aspectos, tales como la identificación y evaluación de elementos críticos sobre factores humanos o el análisis de aquellos aspectos de la cultura organizacional relacionados con la seguridad. Se diseñan entrenamientos específicos para los comandantes de aeronave, centrados en el papel del liderazgo inherente a dicho puesto dentro de la tripulación y comienzan a llevarse a cabo entrenamientos avanzados en CRM dirigidos a aquellos profesionales responsables de la formación y evaluación de factores técnicos y humanos en las compañías aéreas.

Este último aspecto resulta característico de esta tercera eta$\mathrm{pa}$, durante la cual comienza a extenderse el entrenamiento en CRM a otros colectivos de profesionales de la aviación (Helmreich, 2006), como las azafatas de vuelo y el personal de mantenimiento (Taylor y Thomas, 2003), entre otros. Además, los operadores aéreos comienzan a realizar entrenamientos conjuntos de CRM para tripulantes de cabina de vuelo y tripulantes de cabina de pasajeros, de modo que esta tercera generación amplía el concepto de tripulación de vuelo, cubriendo algunas de las lagunas de los programas de la segunda generación.

\section{Cuarta generación: Integración del CRM en las operaciones de vuelo}

En el año 1990, la F.A.A. da un paso más y convierte el CRM en requisito reglamentario para todas las líneas aéreas de los EE.UU. Poco después, en 1994, la U.S. Air Force obliga a todos los componentes de sus tripulaciones de vuelo a recibir entrenamiento y evaluación en CRM (O'Connor, Hahn y Nullmeyer, 2010). Es también al comienzo de esta década cuando la F.A.A. inicia su Programa de Capacitación Avanzada (Advanced Qualification Program [A.Q.P.]) (Birnbach y Longridge, 1993; Helmreich, 2006), que supondrá uno de los mayores cambios en lo referente al entrenamiento de las tripulaciones de vuelo. El A.Q.P. permite crear entrenamientos CRM "a medida", posibilitando que cada operador haga frente a sus necesidades específicas (Salas et al., 2001). Gracias al mismo, los planes de formación se hacen más flexibles y adaptables a las particularidades de cada compañía, e incluso a cada tipo de aeronave, posibilitando una respuesta concreta a los problemas específicos, relacionados con los factores humanos, de cada operador.

Además, como parte de la integración del CRM en las operaciones de vuelo, muchas compañías comienzan a protocolizar conceptos, introduciendo comportamientos específicos a sus listas de verificación (checklists) (Helmreich, 2006). Con ello se pretende que la actuación de cada uno de los miembros de la tripulación esté fijada de antemano, de modo que cada profesional tenga claramente definido qué hacer y cómo hacerlo en cada una de las maniobras a realizar 0 incluso ante aquellos eventos de emergencia que pudiesen presentarse. El objetivo no era otro que el de asegurar que las decisiones fueran tomadas y las acciones realizadas teniendo en cuenta una línea maestra, haciendo que los fundamentos del CRM estuvieran siempre presentes, particularmente en situaciones no habituales (Helmreich et al, 1999; Marshall, 2010). La estandarización de los procedimientos de actuación y listas de verificación, junto con la declarada aceptación de su utilización por parte de las tripulaciones aéreas, han sido, desde los programas de CRM de cuarta generación, un campo de trabajo importante como elemento crítico con el que reducir la siniestralidad aérea.

\section{Quinta generación: Gestión y normalización del error}

Lo fundamental de este periodo es la consideración del CRM como estrategia de gestión y reducción del error (Helmreich, 2006). Los programas de formación tienen ahora como objetivo la normalización del error y el desarrollo de estrategias para la gestión del mismo (Helmreich, 1997). Se parte de la idea de que el error es inevitable (Salas et al., 2001), por lo que el interés se centra en la generación de "barreras" para evitar la aparición del mismo y en la creación de las herramientas necesarias para gestionarlo adecuadamente si se presenta. Se considera que un error bien gestionado es indicador de un desempeño eficaz, adquiriendo una importancia primordial el entrenamiento en habilidades de trabajo en equipo como herramienta para conseguirlo (Salas et al., 2001).

La evitación del error humano ya estaba presente en los primeros programas de entrenamiento en CRM. Sin embargo, su justificación y puesta en práctica no había sido la deseada. Esta etapa se fundamenta en la premisa de que el error humano, además de inevitable, es ubicuo, por lo que hay que convivir con él. Pero además se le considera una valiosa fuente de información. Si el error es inevitable y ubicuo, el CRM se define como un conjunto de contramedidas para frenar dicho error, con tres líneas fundamentales de defensa. La primera, por supuesto, es la evitación de la aparición error; la segunda es la anticipación o búsqueda de errores incipientes antes de que se cometan; y la tercera y última es la mitigación de las consecuencias asociadas a los errores que se han producido y que no hemos sido capaces de evitar. El CRM se convierte así en 
una metodología que incluye un conjunto de contramedidas o estrategias eficaces para resolver con éxito aquellas situaciones que pueden conducir a una aeronave al desastre. La diferencia en cuanto a su modo de utilización radica simplemente en el momento en que se detecta el error.

El CRM de quinta generación hizo hincapié en la recopilación y análisis de datos como método para avanzar en la comprensión de los errores. Con este objetivo, la F.A.A. puso en marcha los Programas de Acción de Seguridad en Aviación (Aviation Safety Action Programs [A.S.A.P.]), iniciativa cuyo objetivo era fomentar la notificación de incidentes dentro de las compañías aéreas, a fin de abordar los problemas de seguridad de una manera proactiva (F.A.A., 1997), y que supuso un éxito rotundo (Helmrich et al, 1999).

\section{Sexta generación: La gestión del error y la amenaza propia del ámbito de operación. Situación actual del CRM}

Los programas de entrenamiento en CRM han continuado su evolución, condicionados sobre todo por los sucesos que han ocurrido en el mundo de la aviación y por el desarrollo de la ciencia psicológica en los últimos años. La relevancia de los entrenamientos en CRM en el contexto operacional es esencial (Maurino, 1999) y, actualmente, la evidencia sobre la efectividad del entrenamiento en CRM, aunque imperfecta, es impresionante (Salas et al., 2001). Siguiendo a Helmreich (2006) y Marshall (2010), podemos afirmar que nos encontramos dentro de lo que ya se conoce como sexta generación de los programas de entrenamiento en CRM, que surge como lógica continuación de la generación anterior y refleja el hecho de que las tripulaciones aéreas ya no sólo deben enfrentarse al error humano dentro de la cabina, sino también gestionar las amenazas externas provenientes del ámbito de operación (Helmreich, 2006). La característica fundamental de esta etapa es que se tiene una conciencia mayor de aquellos riesgos contextuales que deben ser manejados. Las tripulaciones de vuelo deben ahora enfrentarse también a las amenazas a la seguridad de vuelo que surgen del entorno de trabajo en su conjunto, esto es, aquellas que provienen del entorno operativo (por ejemplo, un cálculo erróneo de combustible a la hora de repostar el avión por parte del personal de la aerolínea o un error de comunicación por parte del controlador de tráfico aéreo).

Como consecuencia de esto, el foco de atención ya no está puesto de forma unitaria en el piloto (como en los entrenamientos de primera generación), ni en la tripulación (propio de las generaciones segunda y posteriores), ni sólo en el entrenamiento específico en el uso de la automatización y el papel del liderazgo de los comandantes de aeronave (destacado en la tercera generación), ni siquiera únicamente en el enfoque de la gestión del error (de la quinta generación), que reforzaba el enfoque formativo de la A.Q.P. de la cuarta generación, sino que la gestión de la amenaza y con ello de la seguridad abarca un campo mucho más amplio, aquel en el que se desarrollan las operaciones aéreas.
Por ello, en la actualidad, la metodología CRM tiene como objetivo no sólo eliminar, frenar o mitigar los errores, sino también identificar y hacer frente a las amenazas sistémicas a la seguridad que se presentan en un mundo tan complejo como es el de la aviación. El salto cualitativo que se produce entre la quinta y la sexta generación es la toma de conciencia de un nuevo aspecto, la amenaza externa, que supone nuevos retos $y$ desafíos para la ciencia psicológica aplicada a la aviación.

Sin embargo, y a pesar de los muchos progresos conseguidos, aún queda mucho por hacer, ya que existen diferencias en la formación de unas compañías a otras. A pesar que en el año 1998 el entrenamiento en CRM se convirtió en obligatorio para todas las compañías aéreas del mundo (International Civil Aviation Organization [I.C.A.O.], 1998), hasta hace poco tiempo no todas las tripulaciones de aviación recibían dicha formación (F.A.A., 2012). A esto hay que añadir que existen aspectos importantes que el CRM no incluye y que pueden resultar básicos para ayudar a las tripulaciones en su trabajo y con ello reducir aún más los accidentes aéreos (Muñoz-Marrón, en realización; Muñoz-Marrón, Gil y Lanero, en realización). Por otra parte, si bien los procedimientos de actuación o checklists constituyen una herramienta muy útil y eficaz para aquellas situaciones de vuelo rutinarias o frente a fallos de sistemas o emergencias aéreas de baja peligrosidad, su utilización en situaciones de extrema peligrosidad caracterizadas por su novedad, escasa probabilidad de aparición y carácter imprevisible ha sido cuestionada, ya que puede llevar aparejada cierta pérdida de iniciativa por parte de la tripulación en cuanto a toma de decisiones eficaces se refiere (Muñoz-Marrón, Gil y Lanero, en realización). Quizás por esta razón o por la falta de evidencia para apoyar la idea de que el entrenamiento en CRM está teniendo un efecto directo sobre la tasa de accidentes (O'Connor, Jones, McCauley y Buttrey, 2012), la relación entre el CRM y el aumento de la seguridad aérea resulta tenue (Maurino, 1999) o poco evidente. Esta falta de evidencias viene provocada en gran medida por el reducido tamaño muestral de los diferentes estudios realizados, así como por la pequeña tasa de accidentes aéreos que, afortunadamente, se producen en la actualidad y que ha hecho imposible, hasta el momento, establecer una relación directa entre el entrenamiento en CRM y la disminución de la accidentabilidad aérea.

Cuando hablamos de los programas de entrenamiento en CRM es fácil entender tanto sus contribuciones como sus limitaciones. Lo que es evidente es que la razón fundamental para la formación en factores humanos es tan fuerte ahora, si no más, que cuando el término CRM fue acuñado por primera vez (Helmreich, 1999). Es importante dejar claro que "el CRM no es y nunca será el mecanismo que elimine el error y garantice la seguridad en un entorno de riesgo como la aviación" (Helmreich, et al., 1999, p. 30), del mismo modo que conviene insistir en que constituye en la actualidad la herramienta más potente que poseen los operadores aéreos en lo que a entrenamiento en factores humanos se refiere. Su desarrollo está en 
continua evolución, haciéndose cada vez más completo y sofisticado, integrando aspectos de una industria que avanza con rapidez y ayudándose de las aportaciones que otras ciencias, como la Psicología, ponen a su disposición en el interminable intento de avanzar en el camino de la reducción de los accidentes aéreos. Las inevitables consecuencias de pérdida de vidas humanas que los accidentes aéreos suponen, hacen necesarios continuos esfuerzos por parte de la industria aeronáutica, a los que la Psicología como ciencia no podrá ya nunca ser ajena.

\section{ÁMBITOS DE LA PSICOLOGÍA QUE MÁS HAN CONTRIBUIDO AL DESARROLLO DEL CRM}

Aunque es relativamente fácil identificar aspectos psicológicos en el desarrollo del CRM, ya que son éstos los que conforman su columna vertebral, no lo es tanto aislar la presencia de tales aspectos en cada etapa, ya que los acontecimientos se suceden de una forma no precisamente regular y metódica, y con grandes variaciones dependiendo de los países en los que los programas de entrenamiento se introducen en distintos momentos. Es a posteriori cuando intentamos localizar esos aspectos, para obtener información relevante que, en cierto modo, nos permita hablar de una cierta evolución lógica. Ocurre en todos los procesos, y éste no es una excepción. Intentaremos, sin embargo, extraer de la información que proporciona el proceso del desarrollo y conformación del CRM aquellos elementos que de manera más directa hacen referencia a aspectos en los que, dado su contenido, la última palabra desde el punto de vista científico le corresponde a la ciencia psicológica.

La problemática alrededor de la cual gira el CRM es muy variada, destacando sin duda de manera especial los siguientes temas: liderazgo, gestión de recursos humanos, trabajo en equipo, comunicación, habilidades interpersonales, formación y entrenamiento, toma de decisiones y gestión eficaz del error o desempeño eficaz. Toda esta temática, cuyo objetivo se centra siempre en un intento por alcanzar un rendimiento o desempeño eficaz para evitar el error $y$, consecuentemente, el accidente, tiene su origen en la Psicología Social, precisamente en los trabajos pioneros de Lewin $(1940,1945,1947)$ y colaboradores (Lewin, Lippitt y White, 1939) sobre liderazgo y estudio de la gestión grupal, con toda la problemática interpersonal que conlleva el trabajo en equipo y la necesidad de profundizar en el estudio de las habilidades interpersonales que se ponen en juego cuando se trabaja de este modo. Se hizo mucho hincapié en las dinámicas de grupos (propias de la segunda generación de CRM) y su aplicación en la metodología de formación en el aula o del entrenamiento en situaciones que simularan situaciones reales, con el desarrollo del estudio de casos, el training group (Lewin, 1945) y la técnica del role playing (Maier, Solem y Maier, 1975). La Psicología Social posterior continua profundizando en estos problemas, siendo enriquecida con las contribuciones de la Psicología del Trabajo y de las Organizaciones, conocida históricamente con denominaciones tales como Psicología Industrial, Psicología de los Recursos Humanos, Psicología Ocupacional, Psicología Organizacional, o la denominación más interdisciplinar de Comportamiento Organizacional y Gestión de Recursos Humanos.

Podríamos afirmar, sin duda, que la mayor contribución de la Psicología al mundo de la aviación son los programas de CRM. La incursión de profesionales de la ciencia psicológica en este mundo, no bien recibidos en un primer momento por los pilotos (Helmreich et al., 1999), vino provocada por el objetivo primordial de combatir el error humano. El interés inicial por desarrollar esta faceta, por entonces casi inexplorada en aviación, desde el Ames Research Center de la N.A.S.A. y la aparición de psicólogos como Lauber, Blake, Mouton o el propio Helmreich y su equipo de colaboradores de la Universidad de Texas, provocaron la creación de una rama de la psicología aplicada con un gran potencial futuro: la Psicología Aeronáutica o Psicología de la Aviación.

Dado que el objetivo del CRM está bien identificado y que el proceso va en la dirección esperada de conseguir un mayor nivel de seguridad aérea a partir de una disminución del error humano, es de esperar que la Psicología no deje de tener la importancia que ha tenido hasta el momento. Incluso podríamos suponer, sin el menor atisbo de exageración, que en la investigación futura la contribución de la Psicología será aún mayor, con una incidencia especial en la mejora de la formación de todo el personal de las compañías aéreas (aspecto ya iniciado en la cuarta generación al incluir en los programas de formación a toda la tripulación al completo), y de la selección, formación y entrenamiento de las tripulaciones en materias tales como flexibilidad y creatividad, dada la importancia clave que poseen estas cualidades a la hora de implementar conductas eficaces en situaciones de emergencia y la evidencia de la laguna que se encuentra en la formación actual en tales materias (Muñoz-Marrón, en realización).

\section{CONFLICTO DE INTERESES}

No existe conflicto de intereses.

\section{REFERENCIAS}

Acuña, C. (2013). El entrenamiento CRM (Crew Resource Management) aplicado al ámbito de la Fuerza de Submarinos de la Armada Argentina (Operaciones de Submarinos y de Buzos Tácticos) (Tesis para magister universitario). Universidad Nacional del Mar del Plata, Mar del Plata, Argentina.

Alkov, R. A. (1989). The Naval Safety Center's aircrew coordination training program. En E. Salas, K. A. Wilson y E. Edens (Eds.), Critical essays on human factors in aviation: Crew Resource Management (pp. 3-7). Hampshire, UK: Ashgate.

Alonso, M. M. (1997). Psicología aeronáutica: Desarrollos actuales. En $9^{\circ}$ Congreso Argentino de Psicología, Tucumán, Argentina. 
Amézcua González, L., Lareo, J. y Amézcua Pacheco, O. (2001). Medicina aeronáutica. Madrid, España: Fundación de Estudios y Formación Sanitaria.

Baumgarten, F. (1957). Examen de las aptitudes profesionales. Barcelona, España: Labor.

Birnbach, R. y Longridge, T. (1993). The regulatory perspective. En E. Wiener, B. Kanki, y R. Helmreich (Eds.), Cockpit Resource Management (pp. 263-282). San Diego, CA: Academic Press.

Blake, R. y Mouton, J. (1964). The Managerial Grid: The key to leadership excellence. Houston, TX: Gulf Publishing Co.

Blake, R. y Mouton, J. (1985). The Managerial Grid III: The key to leadership Excellence. Houston, TX: Gulf Publishing Co.

Cooke, N. y Durso, F. (2007). Stories of modern technology. Failures and cognitive engineering successes. Boca Raton, FL: CRC Press.

Cooper, G. E., White, M. D. y Lauber, J. K. (Eds.) (1980). Resource Management on the flightdeck: Proceedings of a NASA Industry Workshop (NASA CP-2120). Moffett Field, CA: NASA-Ames Research Center.

Degani, A., Heymann, M. y Shafto, M. (1999). Formal aspects of procedures: The problem of sequential correctness. Proceedings of the Human Factors and Ergonomics Society Annual Meeting, 43(20), 1113 1117. https://doi.org/10.1177/154193129904302012

Dockeray, F. y Isaacs, S. (1921). Psychological research in aviation in Italy, France, England, and the American Expeditionary Forces. Journal of Comparative Psychology, 1, 115-148.

Federal Aviation Administration (1997). Aviation safety action programs. Advisory Circular 120-66. Washington, DC: Autor.

Federal Aviation Administration (2012). The History of CRM [Video]. Washington, DC: F.A.A. TV.

Flin, R. y Martin, L. (2001). Behavioural markers for CRM: A review of current practice. International Journal of Aviation Psychology, 11, 95-118.

Gordon, S., Mendenhall, P. y O'Connor, B. B. (2013). Beyond the checklist, What else health care can learn from aviation teamwork and safety. New York, NY: Cornell University Press.

Helmreich, R. L. (1997). Managing human error in aviation. Scientific American, 276(5), 62-67.

Helmreich, R. L. (1999). Building safety on the three cultures of aviation. Proceedings of the IATA Human Factors Seminar (pp. 39- 43). Bangkok, Thailand, August 12, 1998.

Helmreich, R. L. (2006). Red alert. Flight Safety Australia, September-October, 24-31.

Helmreich, R. L. y Foushee, H. C. (1993). Why Crew Resource Management? Empirical and theoretical bases of human factors training in aviation. En E. Wiener, B. Kanki y R. Helmreich (Eds.), Cockpit Resource Management (pp. 3-45). San Diego, CA: Academic Press.
Helmreich, R. L., Merritt, A. C. y Wilhelm, J. A. (1999). The evolution of Crew Resource Management training in commercial aviation. International Journal of Aviation Psychology, 9(1), 19-32.

International Civil Aviation Organization (1989). Human Factors Digest №2: Flight crew training: Cockpit Resource Management (CRM) and Line-Oriented Flight Training (LOFT) (Circular 217-AN/132). Montreal, Canada: Autor.

International Civil Aviation Organization (1998). Human factors training manual (Doc. 9683-AN/950). Montreal, Canada: Autor.

Lauber, J. K. (1984). Resource management in the cockpit. Air Line Pilot, 53, 20-30.

Lewin, K. (1940). Studies in topological and vector psychology: 1. Formalization and progress in psychology. University of lowa Studies in Child Welfare 16(3), 9-42.

Lewin, K. (1945). Reserve program of group dynamics. The Research Center for Group Dynamics at Massachusetts Institute of Technology. Sociometry, VIII(2), 126-136.

Lewin, K. (1947). Frontiers in group dynamics: Concept, method and reality in social science; social equilibria and social change. Human Relations, 1(1), 5-41.

Lewin, K., Lippitt, R. y White, K. (1939). Patterns of aggressive behavior in experimentally created social climates. Journal of Social Psychology, 10, 271-299.

Maier, N. R. F., Solem, A. R. y Maier, A. A. (1975). The roleplay technique. A handbook for management and leadership practice. California: University Associates.

Marshall, D. (2010). Crew Resource Management: From patient safety to high reliability. Denver, CO: Safer Healthcare Partners.

Maurino, D. E. (1999). Crew Resource Management: A time for reflection. En D. J. Garland, J. A. Wise y V. D. Hopkin (Eds.), Handbook of aviation human factors (pp. 215-234), Mahwah, NJ: Lawrence Erlbaum Associates.

McGregor, D. (1960). The human side of enterprise. New York, NY: McGraw-Hill.

McKeel, G. (2012). Crew Resource Management. What's Next to SADCLAM? Marine Corps Gazette, 96(5), 44-46.

Muñoz-Marrón, D. (en realización). La Creatividad como componente de la Gestión de Recursos de la Tripulación (CRM) y su influencia en la prevención de los Accidentes Aéreos (Tesis doctoral). Universidad Complutense de Madrid, Madrid, España.

Muñoz-Marrón, D., Gil, F. y Lanero, A. (en realización). Are crews empowered with all the needed resources to successfully address an inflight emergency? Checklist: A necessary but insufficient tool.

National Transportation Safety Board (1978). Aircraft accident report: United Airlines, Inc. McDonnell-Douglas DC-8-81, N8082U Portland, Oregon. December 28, 1978. Washington, DC: United States Government.

O’Connor, P. y Flin, R. (2003). Crew Resource Management 
training for offshore oil production teams. Safety Science, 41, 591-609.

O'Connor, P., Hahn, R. G. y Nullmeyer, R., (2010). The military perspective. En Kanki, B. G., Helmreich, R. L. y Anca, J. (Eds.), Crew Resource Management (pp. 445-466). San Diego, CA: Academic Press.

O'Connor, P., Jones, D., McCauley, M. y Buttrey, S. E. (2012). An evaluation of the effectiveness of the crew resource management program in naval aviation. International Journal of Human Factors and Ergonomics, 1(1), 21-40.

O'Connor, P., Muller, M. (2006). A novel human factors training curriculum for U.S. Navy diving. Paper presented at the Undersea and Hyperbaric Medical Society Meeting, Orlando, FL.

Orlady, H. W. y Foushee, H. C. (1987). Cockpit resource management training (Technical Report NASA CP- 2455). Moffett Field, CA: NASA-Ames Research Center.

Ranter, H. (2016). Aviation safety network. Alexandria, VA: Flight Safety Foundation. Recuperado de https://aviationsafety.net

Ruffell Smith, H. P. (1979). A simulator study of the interaction of pilot workload with errors, vigilance, and decisions (NASA TM-78483). Moffett Field, CA: NASA-Ames Research Center.

Sáiz, M. y Sáiz, D. (2012). El inicio de la psicología aplicada a la aviación: Desde el principio del siglo XX a la década de los años treinta. Revista de Historia de la Psicología, 33(4), 7-36.

Salas, E., Burke, C., Bowers, C. A. y Wilson, K. A. (2001). Te- am training in the skies: Does Crew Resource Management (CRM) training work? Human Factors, 43, 671-674.

Salas, E. y Cannon-Bowers, J. A. (2001). The science of training: A decade of progress. Annual Reviews of Psychology, 52, 471-499. doi: 10.1146/annurev.psych.52.1.471

Salas, E., Fowlkes, J. E., Scout, R. J., Milanovich, D. M. y Prince, C. (1999). Does CRM training improve teamwork skills in the cockpit? Two evaluation studies. Human Factors, 41(2), 326-343.

Salas, E., Wilson, K. A. y Edens, E. (Eds.) (2009). Critical essays on human factors in aviation: Crew Resource Management. Hampshire, UK: Ashgate.

Sebastián, M. L. (2002). Fallo humano: Evitando el error. Líneas del Tren, 278, 22-23.

Sebastián, M. L. (2009). Fallo humano: La quiebra de un paradigma. Apuntes de Psicología, 27(1), 21-51.

Shuffler, M. L., DiazGranados, D. y Salas, E. (2011). There's a science for that: Team development interventions in organizations. Current Directions in Psychological Science, 20, 365-372.

Taylor, J. C. y Thomas, R. L. (2003). Written communication practices as impacted by a maintenance resource management training intervention. Journal of Air Transportation, 8, 69-90.

Turner, T. P. (1995). Cockpit resource management: The private pilot guide. U.S.A.: McGraw-Hill.

Wiener, E. Kanki, B. y Helmreich, R. (Eds.) (1993). Cockpit resource management. San Diego, CA: Academic Press. 